\title{
Metamaterial Inspired Miniaturized SIW Resonator for Sensor Applications
}

\author{
Sen Yan, ${ }^{1,2, a)}$ Juncheng Bao, ${ }^{2)}$ Ilja Ocket, ${ }^{2,3)}$ Bart Nauwelaers ${ }^{2)}$, and Guy. A. E. Vandenbosch ${ }^{2)}$ \\ ${ }^{1}$ School of Electronics and Information Engineering, Xi'an Jiaotong University, Xi'an, Shaanxi, 710049, China \\ ${ }^{2}$ Deptartment of Electrical Engineering, KU Leuven, Kasteelpark Arenberg 10, Box 2444, 3001 Leuven, Belgium \\ ${ }^{3}$ Interuniversity Microelectronics Center (IMEC), Kapeldreef 75, 3001 Leuven, Belgium
}

\begin{abstract}
A planar substrate integrated waveguide (SIW) resonator is proposed in this letter. By loading a metamaterial inspired structure in a short section of an SIW, a miniaturized cavity $\left(0.263 \times 0.184 \times 0.0063 \lambda_{0}^{3}\right.$ at $\left.2.39 \mathrm{GHz}\right)$ with high quality factor $(272.5)$ is obtained. The resonant modes of the cavity and their corresponding field distributions are analyzed. A resonator prototype has been fabricated and tested. The measured results agree well with the simulated ones. This cavity resonator has huge potential in sensor applications. A binary solvent water alcohol mixture is used to verify the sensor concept.
\end{abstract}

\section{Introduction}

Cavity resonators have huge application potential in the microwave and millimeter wave frequency range, e.g. for filters, oscillators, antennas, sensors, etc. To reduce the size of traditional metallic waveguide cavities and simplify the integration with other planar microwave circuits, recently substrate integrated waveguides (SIW) have been widely studied. These waveguides can be fabricated with standard printed circuit board (PCB) technology [1], [2], which will significantly reduce fabrication cost compared to the traditional metallic waveguides. Due to the relatively low radiation loss and high quality factor (Q factor) compared to other planar resonators, SIW cavities are prime candidates to be used in sensor applications [3]-[9].

Similar as for the traditional metallic waveguide cavity, the basic mode of a SIW cavity is also $\mathrm{TE}_{101}$. Its resonant frequency is determined by the permittivity of the loaded material (substrate) and the dimensions of the cavity. To reduce the size of SIW cavities, the epsilon-near-zero (ENZ) tunneling phenomenon [6], [7] can be used. The resonant frequency of ENZ tunneling is just as the cutoff frequency of the $\mathrm{TE}_{10}$ mode of the waveguide, which means that the related operating frequency is independent of the length of the waveguide. Thus, the resonance can be expressed as a $\mathrm{TE}_{100}$ mode. However, this mode cannot be used to further reduce the width of the SIW cavity. Recently, the study of metamaterials has revealed that a waveguide can operate at negative modes if the loaded metamaterial simultaneously has a negative permittivity and negative permeability [10]-[12]. These negative modes can operate much lower than the cutoff frequency of the dominant $\left(\mathrm{TE}_{10}\right)$ mode. The negative

\footnotetext{
a) Corresponding author: Sen Yan, Email: sen.yan@xjtu.edu.cn.
} 
permittivity can be automatically obtained by operating the waveguide below the cutoff frequency of the dominant TE mode. The negative permeability can be realized by inserting certain slots on the surface of the waveguide [10].

In this letter, a miniaturized SIW resonator is proposed. By loading two interdigital capacitors (meander slots) on the bottom layer of the SIW, a negative mode $\mathrm{TE}_{10(-1)}$ mode is obtained. It shows a much lower resonant frequency than the normal positive modes. The field distributions in the cavity are analyzed to study the operating mechanism. A prototype is fabricated and measured. The results show that this resonator can be used as a sensor to measure the permittivity of a liquid.

\section{Topology}

The proposed SIW cavity is fabricated using the substrate Rogers RT5880 with thickness $0.787 \mathrm{~mm}$. The dielectric constant and loss tangent are 2.2 and 0.0009 , respectively. The diameter of the vias is $1.2 \mathrm{~mm}$ and the distance between the centers of neighboring vias is $2.5 \mathrm{~mm}$, which guarantees that the leaky wave from the short slots can be neglected. [1]

The topology of the resonator is shown in Fig. 1. A section of SIW consists of two units, and each unit is loaded by an interdigital capacitor (meander slot) on the surface of the SIW. A cavity with size $33 \times 23 \mathrm{~mm}^{2}$ is obtained by shorting the SIW at the two boundaries (left and right). This cavity is fed by two microstrip lines with characteristic impedance $50 \Omega$. The liquid sample is located in a center hole through the cavity. The bottom of the hole is sealed with silica gel. The top of the hole is open in order to be able to inject fluid. The detailed dimensions are labeled in Fig. 1 (a) and (b). The frequency domain solver in the commercial full-wave simulation software CST microwave Studio, which is based on the finite element method, is used in this letter [13].

\section{Operating Mechanism}

Firstly, a unit of SIW is calculated with and without the slot loading, respectively. The dispersion curve of this SIW is then retrieved from the S-parameters, see Fig. 2 (a). When there is no slot loaded on the SIW, the cutoff frequency of the dominant mode $\left(\mathrm{TE}_{10}\right)$ is $5.05 \mathrm{GHz}$. For the proposed structure with slot, the SIW shows a left-handed transmission characteristic (phase advanced section) below the frequency $2.98 \mathrm{GHz}$, and a right-handed transmission characteristic (phase delayed section) above the frequency $3.75 \mathrm{GHz}[10]$. A stop band is observed between these two frequencies.

Next, the cavity is Fig. 1 is analyzed. The resonant frequency of the cavity is determined by the length of the SIW,

$$
m \beta p=n \pi
$$

where $\beta \mathrm{p}$ is the phase delay of one unit cell, $\mathrm{m}$ is the number of unit cells ( 2 in our structure), $\mathrm{n}$ is the mode number (the number of standing wave periods inside the SIW), which means that $\mathrm{n}$ is an integer $(0, \pm 1, \pm 2, \ldots, \pm(\mathrm{m}-1))$. 
Fig. 2 (b) shows the simulated S parameters of the cavity. When the cavity is without loading slots, its first resonance $\left(\mathrm{TE}_{101}\right)$ is located at $6.544 \mathrm{GHz}$, which agrees well with the dispersion curve in Fig. 2 (a). The slight frequency shift is caused by the feeding line, which may perturb the original field distribution in the cavity. The loading of the slots may decrease the resonant frequency of the $\mathrm{TE}_{101}$ mode to $4.831 \mathrm{GHz}$, and more importantly, two additional lower modes are obtained. One is the negative mode $\mathrm{TE}_{10(-1)}$ located at $2.39 \mathrm{GHz}$, and the other is the zero-order resonance (ZOR) mode at $3.072 \mathrm{GHz}$. The field distributions of these two modes are shown in Fig. 3. The mode $\mathrm{TE}_{10(-1)}$ has one standing wave in the SIW (see Fig. 3 (a) and (b)), similarly to the field distribution of mode $\mathrm{TE}_{101}$, while the magnetic field of the ZOR mode is almost constant in the cavity (see Fig. $3($ d)).
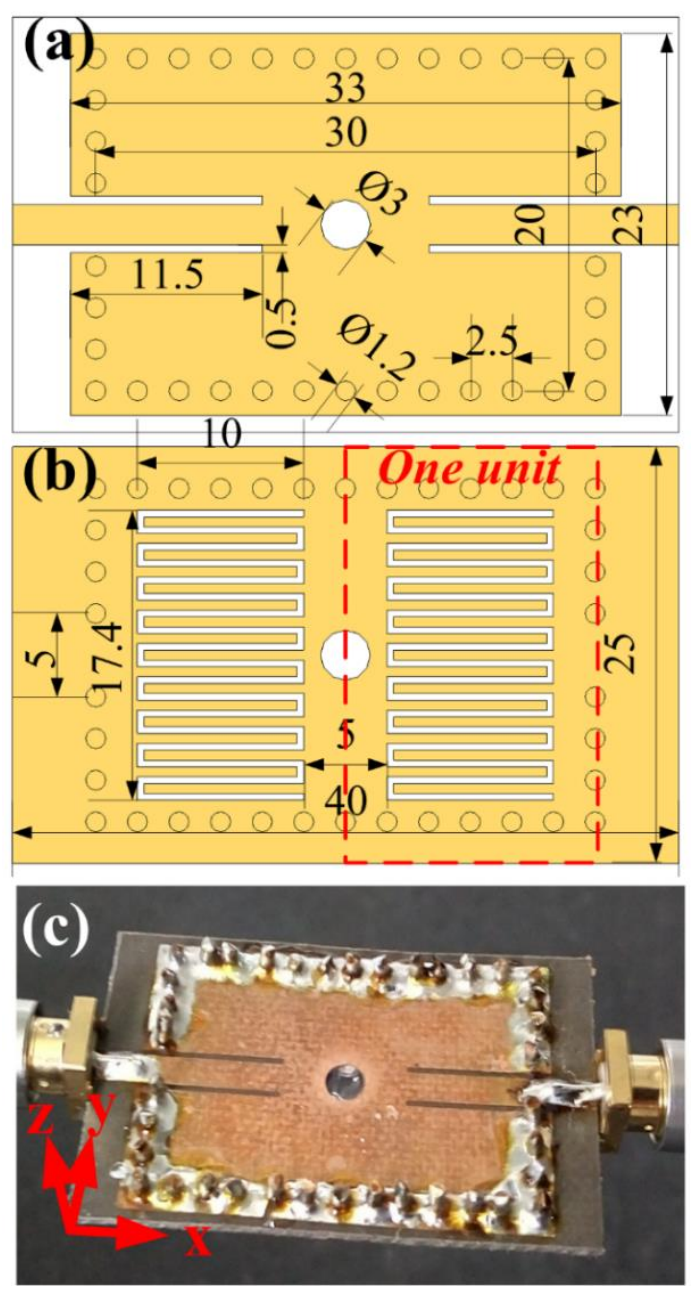

FIG. 1. Topology of the proposed SIW resonator. (a) top layer, (b) bottom layer, (c) photo of the fabricated prototype. The widths of the fingers and gaps are $0.6 \mathrm{~mm}$ and $0.4 \mathrm{~mm}$, respectively. 


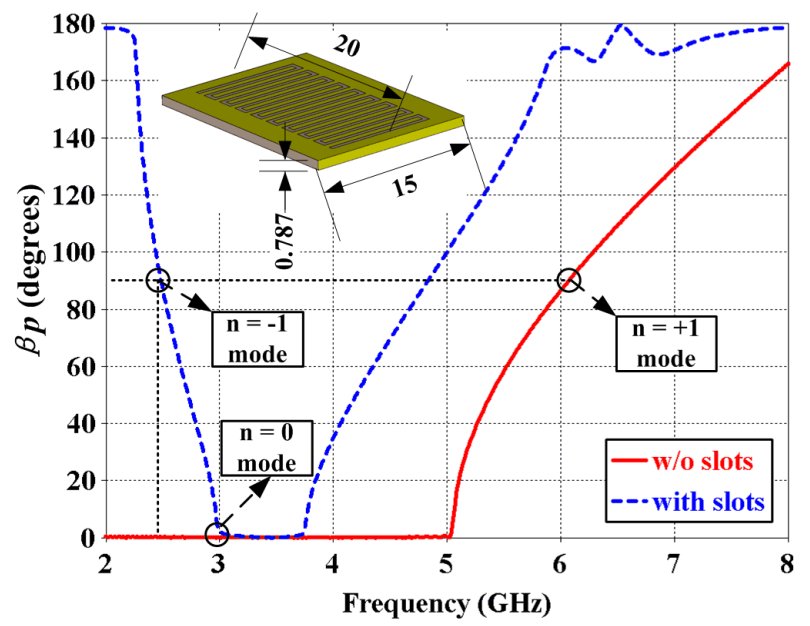

(a)

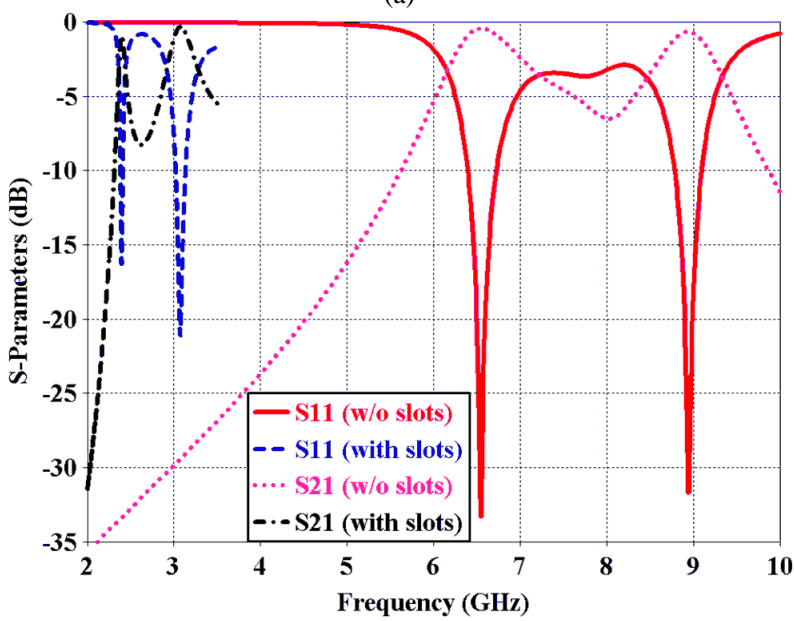

(b)

FIG. 2. (a) Dispersion curve of the SIWs with and without slot loading, (b) S parameters of the cavity with and without slot loading. The analysis of the topology with slot is based on a single cell.

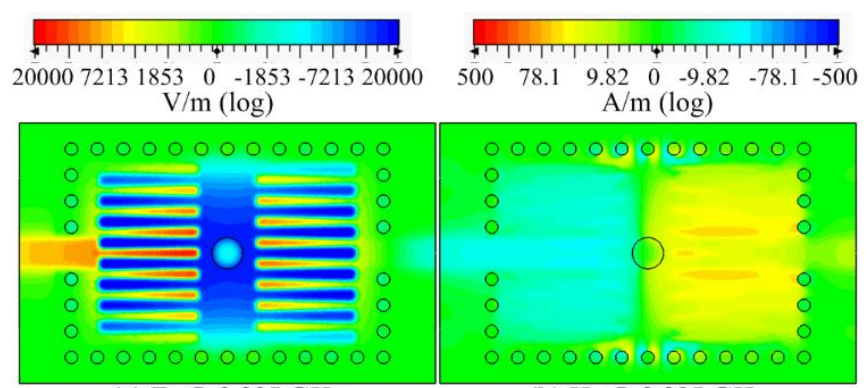

(a) $\mathrm{E}_{\mathrm{z}} @ 2.395 \mathrm{GHz}$

(b) $\mathrm{H}_{\mathbf{y}}$ (a) $2.395 \mathrm{GHz}$

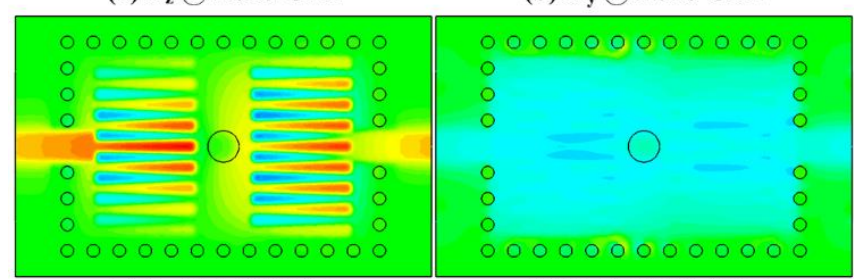

(c) $\mathbf{E}_{\mathrm{z}}$ (a) 3.072 GHz

(d) $\mathrm{H}_{\mathrm{y}} @ 3.072 \mathrm{GHz}$

FIG. 3. Field distributions in the proposed resonator. The cavity is fed from the left port and the coordinate system is defined in Fig. 1. 


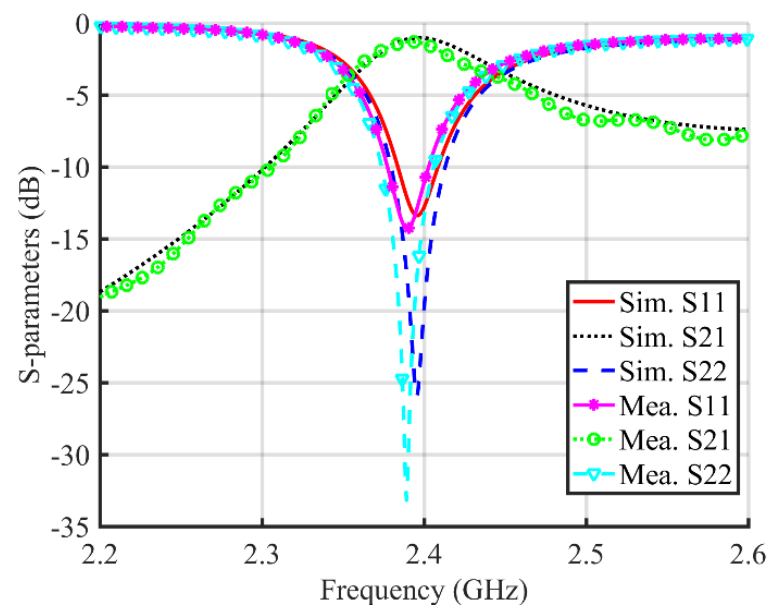

FIG. 4. Simulated and measured S parameters without liquid.
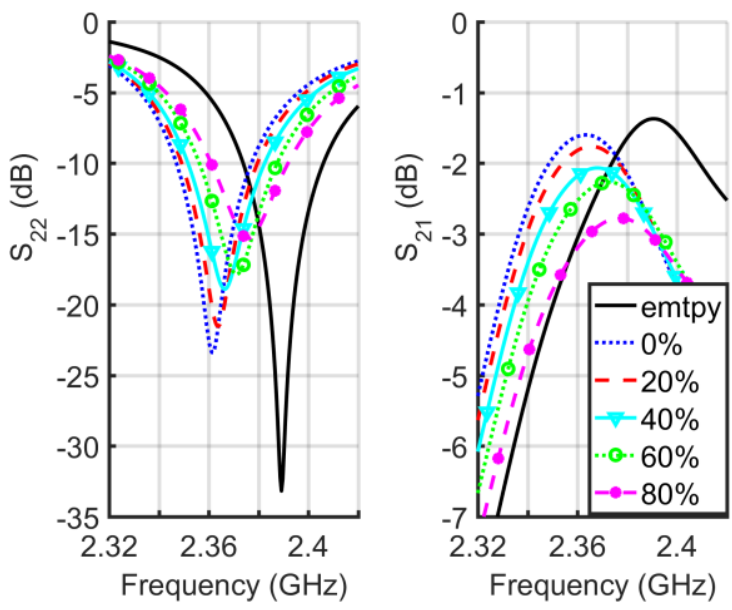

FIG. 5. Measured S parameters with liquid.

In this design, the $\mathrm{TE}_{10(-1)}$ is chosen as the operating mode, not only because it operates at a lower resonant frequency than the ZOR mode, but more importantly, also because the electric field distribution is more concentrated around the center of the cavity. The relatively high field intensity results in the high sensitivity necessary in sensor applications. It should be noted that although the electric field is more concentrated in the meandering slot than at the center of the cavity, the latter location is chosen in our proposed design since it is easy to integrate with a vertical liquid tube.

When a liquid sample is located in the center hole of the cavity, it will couple with the electric field, and thus perturb the resonant frequencies. As for the normal $\mathrm{TM}_{101}$ mode in the waveguide cavity, the frequency shift of the $\mathrm{TE}_{10(-1)}$ mode is also approximately proportional to the dielectric constant of the sample, since the perturbed electric field will be stronger when the dielectric constant of the sample is higher. 


\section{Measurement and Results}

A prototype of the cavity resonator was fabricated, as shown in Fig. 1 (c). The measured S parameters agree quite well with the simulated ones, see Fig. 4. A sharp resonance is observed at ca. $2.39 \mathrm{GHz}$, with a $\mathrm{Q}$ factor of 272.5. The $\mathrm{Q}$ factor is calculated from the input impedance of the left port when the right port of the cavity is shorted [14].

Next, a water alcohol mixture was tested with this resonator. Fig. 5 shows the measured S-parameters. Both the frequency shift of the resonance and the magnitude of the transmission $\left(S_{12}\right)$ show a good monotonicity with a growing alcohol concentration. A further analysis on parameter retrieval reveals that the figure-of merit (FOM) is ca.

$$
F O M=\frac{\Delta f}{\Delta \varepsilon_{r}^{\prime}}=0.34 M H z
$$

where $\Delta \mathrm{f}$ and $\Delta \varepsilon \mathrm{r}^{\prime}$ are the frequency shift and the permittivity shift corresponding with the water alcohol mixed sample [15], respectively. Other parameter retrieval methods for microfluidic sensors can be found in [16] and [17].

The experiment was repeated 5 times for deionized water, and we found that the results are stable. The maximum shift of the resonant frequency was less than $1 \mathrm{MHz}$. It should be mentioned that the major limitation on the accuracy is the knowledge of the exact volume of the liquid. In this preliminary test, the volume of the liquid was controlled by using a pipette. This can be improved by using other methods, e.g., digital pumps.

\section{Conclusion}

A compact SIW cavity resonator was proposed in this letter. By loading a metamaterial inspired structure in the cavity, the resonator can operate at its negative mode, which has a much lower resonant frequency than the traditional dominant mode. Field distributions were analyzed to study the operating mechanism of the cavity. A prototype was fabricated and measured, yielding a good agreement with simulated results. An initial test has shown that the cavity can be used as a permittivity sensor to characterize samples of liquid.

Acknowledgments: This work was partly supported by the Research Foundation-Flanders (FWO) Postdoctoral Fellowship (No. $12 \mathrm{O} 1217 \mathrm{~N})$.

\section{REFERENCES}


[1] M. Bozzi, A. Georgiadis and K. Wu, "Review of substrate-integrated waveguide circuits and antennas," IET Microwaves, Antennas \& Propagation, vol. 5, no. 8, pp. 909-920, June 62011.

[2] K. Wu, Y. J. Cheng, T. Djerafi and W. Hong, "Substrate-Integrated Millimeter-Wave and Terahertz Antenna Technology," Proceedings of the IEEE, vol. 100, no. 7, pp. 2219-2232, July 2012.

[3] J. D. Barrera, and G. H. Huff, “Analysis of a variable SIW resonator enabled by dielectric material perturbations and applications," IEEE Trans. Microw. Theory Techn., vol. 61, no. 1, pp.225-233, Jan. 2013.

[4] H. El Matbouly, N. Boubekeur and F. Domingue, "Passive Microwave Substrate Integrated Cavity Resonator for Humidity Sensing," IEEE Trans. Microw. Theory Techn., vol. 63, no. 12, pp. 4150-4156, Dec. 2015.

[5] C. Liu, and F. Tong, "An SIW Resonator Sensor for Liquid Permittivity Measurements at C Band,” IEEE Microw. Wireless Compon. Lett., vol. 25, no. 11, pp. 751-753, Nov. 2015.

[6] H. Lobato-Morales, A. Corona-Chávez, J. L. Olvera-Cervantes, R. A. Chávez-Pérez, and J. L. Medina-Monroy, “Wireless Sensing of Complex Dielectric Permittivity of Liquids Based on the RFID," IEEE Trans. Microw. Theory Techn., vol. 62, no. 9, pp. 2160-2167, Sep. 2014.

[7] A. K. Jha, and M. J. Akhtar, "Design of Multilayered Epsilon-Near-Zero Microwave Planar Sensor for Testing of Dispersive Materials," IEEE Trans. Microw. Theory Techn., vol. 63, no. 8, pp. 2418-2426, Aug. 2015.

[8] I.-J. Hyeon, W.-Y. Park, S. Lim, C.-W. Baek, "Ku-band bandpass filters using novel micromachined substrate integrated Waveguide structure with embedded silicon vias in benzocyclobutene dielectrics," Sensors and Actuators A: Physical, vol. 188, pp. 463-470, 2012.

[9] T. Yun, S. Lim, "High-Q and miniaturized complementary split ring resonator-loaded Substrate integrated waveguide microwave sensor for crack detection in metallic materials," Sensors and Actuators A: Physical, vol. 214, pp. 25-30, 2014.

[10] Y. Dong, and T. Itoh, "Promising future of metamaterials,” IEEE Microw. Mag., vol. 13, no. 2, pp.39-56, Mar. 2012.

[11] S. Yan, P. J. Soh, G. A. E. Vandenbosch, "Wearable dual-band composite right/ left-handed waveguide textile antenna for WLAN applications," Electron. Lett., vol. 50, no. 6, pp. 424-426, Mar. 2014.

[12] Y. Dong and T. Itoh, "Miniaturized Substrate Integrated Waveguide Slot Antennas Based on Negative Order Resonance," IEEE Transactions on Antennas and Propagation, vol. 58, no. 12, pp. 3856-3864, Dec. 2010.

[13]Computer Simulation Technology (CST). (2016). Microwave Studio. [Online]. Available: https://www.cst.com/Products/CSTMWS

[14] T. Ohira, "What in the World Is Q? [Distinguished Microwave Lecture]," IEEE Microwave Magazine, vol. 17, no. 6, pp. 42-49, June 2016. 
[15]A. Megriche, A. Belhadj, A. Mgaidi, "Microwave dielectric properties of binary solvent water-alcohol, alcohol-alcohol mixtures at temperatures between $-35^{\circ} \mathrm{C}$ and $+35^{\circ} \mathrm{C}$ and dielectric relaxation studies," Mediterranean Journal of Chemistry, vol. 1, no. 4, pp. 200-209, 2012.

[16] A. Ebrahimi, W. Withayachumnankul, S. Al-Sarawi and D. Abbott, "High-Sensitivity Metamaterial-Inspired Sensor for Microfluidic Dielectric Characterization," IEEE Sensors Journal, vol. 14, no. 5, pp. 1345-1351, May 2014. doi: 10.1109/JSEN.2013.2295312

[17] W. Withayachumnankul, K. Jaruwongrungsee, A. Tuantranont, C. Fumeaux, and D. Abbott, "Metamaterial-based microfluidic sensor for dielectric characterization," Sens. Actuators A, Phys., vol. 189, pp. 233-237, Jan. 2013. 